\title{
FUNCIÓN DE LA CORONA EN PASSIFLORA CAERULEA (PASSIFLORACEAE) COMO ATRAYENTE DE SUS VECTORES DE POLINIZACIÓN
}

\author{
GONZALO VARELA ${ }^{1}$, ANDREA COCUCCl${ }^{2}$ y ALICIA N. SÉRSIC²
}

\begin{abstract}
Summary: Role of the corona in Passiflora caerulea (Passifloraceae) as attractant to their pollinators. Passiflora caerulea L. flowers, like those of many other zoophilic species, offer nectar as reward to their pollinators as a primary attractant. This species has developed stimuli or secondary attractants that announce the presence of primary one (i.e. the nectar) and ensure repeated visits from their pollination vectors optimizing reproduction. The corona, a characteristic structure of the Passifloraceae family, has been reported for $P$. caerulea as the organ that produces visual and olfactory stimuli on their pollinators. By preference experiments, nullifying either one or both functions, the importance of each one in attracting pollinators was evaluated. The suppression of functions, individually and together, produced a significant decrease in the number of visits compared to the controls. Thus, the importance of visual and odorant functions was verified; greater efficacy was recorded for the last one in the frequency of visits. Additionally, the volatile compounds produced by the flowers were identified, some of which were produced by the perianth and not by the corona, as was expected.
\end{abstract}

Key words: Passiflora caerulea, corona, secondary attractants, visual function, odorant function, pollination.

\begin{abstract}
Resumen: Las flores de Passiflora caerulea L., como las de muchas otras especies zoófilas, ofrecen néctar como recompensa a sus agentes de polinización a modo de atrayente primario. Esta especie ha desarrollado estímulos, o atrayentes secundarios, que anuncian la presencia de los primarios y que garantizan las visitas repetidas de los vectores de polinización optimizando su reproducción. En $P$. caerulea, la corona, una estructura característica de la familia, ha sido citada como estructura productora de aromas atrayentes y, en conjunto con otras piezas florales, estaría encargada de producir estímulos visuales y olfativos sobre sus polinizadores. Mediante experimentos de preferencia, anulando una o ambas funciones, se determinó la importancia de cada una de ellas. La supresión de las funciones, tanto de manera individual como en conjunto, produjo una disminución significativa en la cantidad de visitas con respecto a los controles. De esta manera, se verificó tanto la importancia de la función visual como odorífera, aunque se registró mayor efectividad de esta última en cuanto a la frecuencia de visitas. Adicionalmente, se identificaron los compuestos volátiles producidos por las flores, algunos de los cuales fueron, sorprendentemente, producidos por el perianto y no por la corona como se esperaba.
\end{abstract}

Palabras clave: Passiflora caerulea, corona, atrayentes secundarios, función visual, función olfativa, polinización.

\section{INTRODUCCIÓN}

Las flores polinizadas por animales (zoofilia) generalmente ofrecen polen o néctar como

${ }^{1}$ Facultad de Ciencias Exactas Físicas y Naturales. Universidad Nacional de Córdoba.

${ }^{2}$ Laboratorio de Ecología Evolutiva y Biología Floral. Instituto Multidisciplinario de Biología Vegetal-CONICET. Facultad de Ciencias Exactas, Físicas y Naturales. Universidad Nacional de Córdoba. recompensa primaria a sus agentes polinizadores para garantizar su visita repetida. Como los atrayentes primarios generalmente no evocan por sí mismos una conducta de visita, los polinizadores son atraídos por atrayentes secundarios: una serie de estímulos visuales, olfativos y táctiles que en conjunto producen la atracción del visitante y determinan su comportamiento, de acuerdo con su percepción sensorial, habilidad de aprender y experiencia (Dafni, 1992). Según el "principio del polinizador más eficiente" propuesto por Stebbins 
(1970), son aquellos polinizadores que ejercen una presión selectiva sobre caracteres de atracción de las flores de modo que producen un aumento del éxito reproductivo de la planta (Olsen, 1997); así, un rasgo floral es considerado una adaptación cuando ocurre un proceso de selección natural mediado por dicho polinizador (Gómez, 2002). De esta manera, especies polinizadoras relacionadas en cuanto a su morfología o comportamiento ejercerán presiones selectivas similares sobre los rasgos florales de las especies de plantas, que se traducirán en una convergencia de esos rasgos hacia ciertos patrones distintivos o "síndromes de polinización" (Faegri \& Van der Pijl, 1979) para ese grupo de polinizadores.

El género Passiflora L. incluye más de 500 especies (Dhawan et al., 2004), distribuidas ampliamente en los trópicos de América, y unas pocas en Asia y Oceanía (Yockteng \& Nadot, 2004), constituyendo el género más numeroso de la familia Passifloraceae (Maout \& Decaisne, 1876; Rendle, 1959; Hickey \& King, 1988; Ulmer \& MacDougal, 2004). Comprende arbustos y hierbas, en su mayoría trepadoras con zarcillos axilares, de tallos herbáceos o leñosos, trepadores y raramente arborescentes (Dhawan et al., 2004). Sus flores son perfectas, con cinco pétalos y cinco sépalos de gran variedad de colores y tamaños dentro de la familia (Killip, 1938; MacDougal, 1994) y un ciclo de brácteas en la base. Los verticilos fértiles están usualmente elevados en relación a las piezas estériles mediante un androginóforo, se componen de cinco estambres alternipétalos y un ovario de placentación parietal formado por tres carpelos y un lóculo; el gineceo termina en tres ramas estilares. El fruto suele ser una baya, a veces comestible como en el caso del maracuyá, $P$. edulis Sims.

Un rasgo distintivo de la familia es una estructura especial en la región más interna del perianto denominada corona, la cual está formada por distintos tipos de piezas en distintos ciclos, cuyas características varían según la especie. Lindman (1906), Puri (1948) y Cusset (1967) han designado con el nombre de Radii a los filamentos externos y Pali a los internos; ambos verticilos pueden presentarse en una o más series, siendo los externos más vistosos que los internos en cuanto a coloración y tamaño. En una posición más interna se encuentra el Operculum, una membrana que se inclina directamente (liso) o formando pliegues (plicativo) hacia el androginóforo (Deginani, 2001) y que protege el paso hacia el anillo nectarífero, ubicado por debajo del mismo. Desde la base del androginóforo y llegando hasta el Operculum se encuentra el Limen, otro ciclo que ayuda a proteger y cubrir el anillo nectarífero. La presencia y morfología de la corona varía extensamente dentro del género Passiflora (Hemingway et al., 2011) y según Janzen (1968), la disposición especial de estas piezas es uno de los factores que determina el gremio de los polinizadores y, por extensión, favorece la polinización cruzada en el género (Knight \& Winters, 1962).

La función de esta estructura varía según el ciclo del que se trate. En muchos casos, mientras los ciclos más internos de la corona cumplen la función de proteger el nectario, los ciclos más externos sirven como atrayentes de los polinizadores, tanto de manera visual como odorífera (Hemingway et al., 2011) o como sitio de aterrizaje para los agentes de polinización en especies melitófilas (Amela García, 1999). En Passiflora caerulea, la estructura anatómica y la ultra-estructura de los filamentos de la corona denotan su carácter secretor como otra forma de atracción de sus agentes polinizadores además de los patrones visuales (Amela García et $a l ., 2007) \mathrm{y}$, aunque en otras especies el tamaño de esta estructura se ha relacionado con la cantidad de benzenoides producidos (Lindberg et al., 2000), no se ha verificado el efecto que produce la corona de $P$. caerulea en el comportamiento de los insectos polinizadores, tanto por su carácter odorífero como visual.

Este trabajo propone comprobar el rol adaptativo de la corona en $P$. caerulea como atrayente de sus agentes de polinización, anulando de manera separada y en conjunto las funciones visual y odorífera y registrando la incidencia de los tratamientos sobre la frecuencia de visitas florales, esperando una disminución cuando dichas funciones son suprimidas.

Cabe aclarar que si bien la corona es una estructura característica de Passifloraceae, no es exclusiva de ella y ha evolucionado de manera independiente en varias ocasiones en diversas familias (Stevens, 2001; Endress \& Matthews, 2006) con distintas funciones, como por ejemplo en el género Narcissus L. (Amaryllidaceae) que funciona como osmóforo (Vogel, 1990; Dobson et al., 1997) y sitio de aterrizaje (Hodgins \& Barrett, 
2008) o en Araujia odorata (Hook. \& Arn.) Fontella $\&$ Goyder (Apocynaceae) que funciona protegiendo el nectario y acumulando el néctar (Galetto, 2006); adicionalmente, el origen morfológico de esta estructura no siempre es el mismo en todos los linajes donde aparece.

\section{Materiales y Métodos}

P. caerulea es una liana de tallo glabro y verdoso, posee hojas simples, pecioladas, de láminas 3, 5 o 6 palmatipartidas, papiráceas o coriáceas y de borde entero, con glándulas tanto en el pecíolo como en la lámina y zarcillos con torsiones apicales que le permiten trepar sobre árboles, arbustos, rejas y tapias. Posee además estípulas en forma de riñón y un verticilo de brácteas en el ápice del pedúnculo. Las flores (Fig.1,A) son de entre 40 y 90 mm de diámetro, con un hipanto de 4-8 $\mathrm{mm}$ de longitud, sépalos de 22-32 mm de longitud por 9-13 $\mathrm{mm}$ de ancho, color verde brillante y pétalos de $16-33 \mathrm{~mm}$ de longitud por $9-12 \mathrm{~mm}$ de ancho, color blanco-verdoso; despiden un fuerte aroma a piretro (Deginani, 2001) y permanecen abiertas generalmente un día, a veces durante la noche, mientras que en días nublados la antesis se prolonga (Amela García \& Hoc, 1997).

La corona de $P$. caerulea (Fig.1, A y B) consta de dos series de radii: lineares, alternos y radiados, de 10-16 mm de longitud por 0,2-0,8 de ancho, de color azulado en el ápice, blanco en la región media y morado en la base; y dos series de pali: capilares, levemente capitados, de 0,8-2,2 mm de longitud y de color morado. El operculum es membranáceo, filamentoso en la porción superior, con filamentos morados sub-erectos inclinados hacia el androginóforo, mientras que en la porción inferior es entero, liso y sin filamentos (Deginani, 2001). El limen es de color blanco, tiene forma de copa y se encuentra rodeando la base del androginóforo. El annulus es un anillo membranoso recurvado debajo del limen que encierra la cavidad nectarífera. La coloración descripta anteriormente es la más habitual, pero pueden existir variaciones dentro de la especie (Amela García, 2013).

Amela García \& Hoc (1997) han descripto tres fases florales para Passiflora caerulea. Durante la fase 1 (donante de polen o fase estaminada) los estigmas se encuentran por encima de las anteras, éstas se hallan dehiscentes y el perianto se dispone de manera perpendicular al eje floral. Durante la fase 2 (donante y receptor de polen o fase estaminadacarpelada) uno o los tres estigmas se encuentran al nivel de las anteras o por debajo de éste, y el perianto sigue en posición perpendicular al eje floral. Durante la fase 3 (donante de polen o fase estaminada) los estilos y estigmas se levantan, las anteras descienden, y el perianto asciende.

Para estandarizar las muestras se trabajó tanto en el campo como en los trabajos de laboratorio exclusivamente con flores en fase 2 , durante la cual la emisión de aromas es más intensa (Amela García et al., 2007).

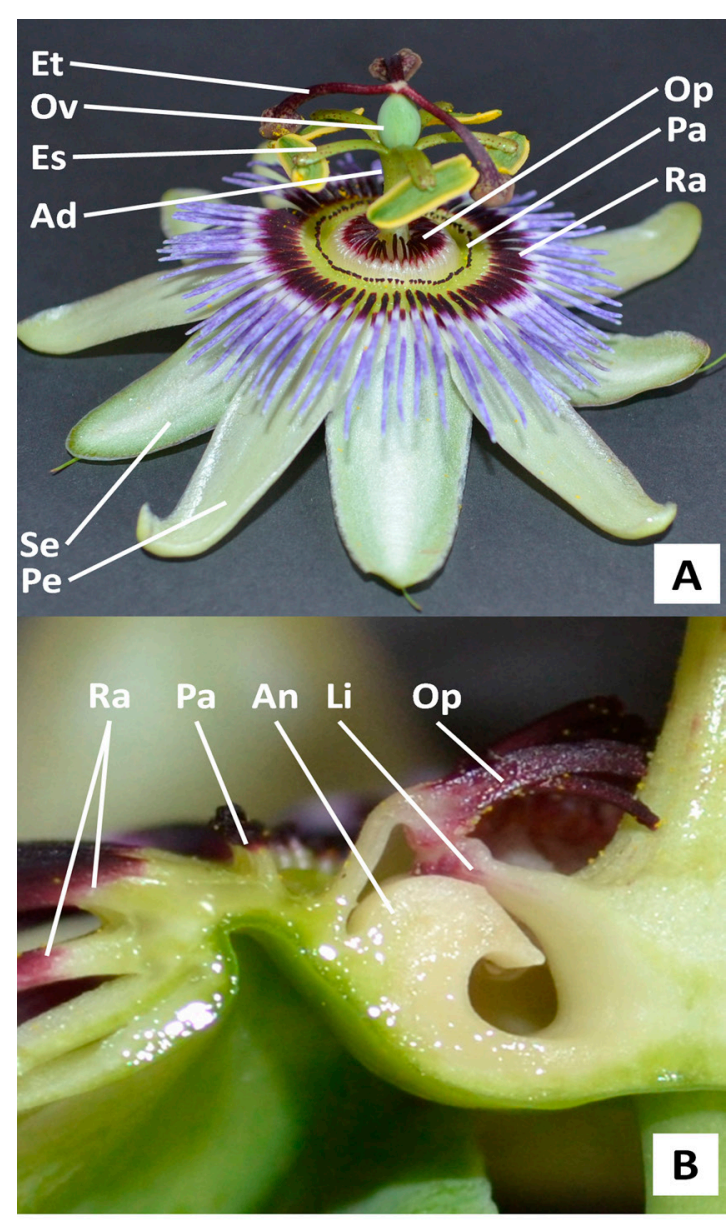

Fig. 1. A: Aspecto general de una flor de P. caerulea. B: Detalle de corte longitudinal mostrando las estructuras que forman la corona. Abreviaciones: Androginóforo (Ad), Annulus (An), Estambre (Es), Estilo (Et), Limen (Li), Operculum (Op), Ovario (Ov), Pali (Pa), Pétalo (Pe), Radii (Ra), Sépalo (Se). 
Para esta especie han sido citados como polinizadores frecuentes Xylocopa augusti, X. frontalis, $X$. nigrocincta, $X$. artifex (Amela García \& Hoc, 1997) así como también Bombus opifex, Bombus morio y Xylocopa splendidula (Torres et al., 2012), aunque también se han observado abejas de la miel, Apis mellifera, visitando las flores y ocasionalmente tocando los órganos fértiles de las mismas.

Los muestreos se realizaron entre los meses de Octubre-Febrero del 2012 y Setiembre-Noviembre de 2013, en poblaciones de $P$. caerulea localizadas en los alrededores de la ciudad de Córdoba, incluyendo la Reserva Hídrica "La Quebrada" en Río Ceballos, domicilios en la misma localidad y en zonas rurales en las Sierras chicas próximas a Carlos Paz. Los muestreos se realizaron entre las 11:00 am y las 17:00 pm, horarios en los cuales se encontraron las flores en fase 2 y visitadas activamente por abejas y abejorros.

Experiencias sobre la emisión de aromas florales y el efecto supresor del aceite en su volatilización:

Se consideró a los radii como la estructura de la corona encargada de producir compuestos volátiles, ya que fueron citados por Amela García et al. (2007) como posible estructura secretora, basándose en su estructura anatómica y ultra-estructura.

Se estudió la identidad de los aromas volátiles secretados por el perianto y por los radii, utilizando un cromatógrafo de gases acoplado a un espectrómetro de masa. Para anular la volatilización de los compuestos producidos en la corona, requisito para uno de los tratamientos de preferencia posteriores, se trabajó con aceite mineral (vaselina liquida, 80\%), cuyos efectos fueron analizados también mediante cromatografía y espectrofotometría de reflexión.

Para analizar la emisión de compuestos de los radii y el efecto supresor del aceite mineral en la emisión de dichos compuestos se procedió a armar una serie de 5 frascos de vidrio transparente de $500 \mathrm{ml}$ de capacidad con distintas porciones de las flores y los respectivos controles: frasco 1: Perianto sin tratar, frasco 2: Radii sin tratar, frasco 3: Radii cubiertos con aceite mineral, frasco 4: Control aceite, frasco 5: Control ambiental, es decir solo aire, sin ninguna pieza floral. Los distintos frascos se cerraron con bolsas para horno (Poliéster TSP02, un PET biorientado) y se dejaron toda la noche de modo que el aire dentro de los frascos se saturara con los aromas emitidos. Una vez transcurrido ese tiempo se expuso en cada muestra la fibra de microextracción 'stableflex' de fase sólida (SPME: Supelco Co., PA, USA, cubierta con divinilbenzeno y polidimetilsiloxano de 100 micras de espesor (Zhang \& Pawliszyn, 1993) por 10 minutos y se inyectaron en un cromatógrafo de gases acoplado a un espectrómetro de masa (GC-MS) para realizar las lecturas.

Se usó un cromatógrafo Clarus 600 de Perkin Elmer perteneciente al INFICQ-IMBIV (CONICETUNC). El software Turbo Mass fue utilizado para adquirir la información del GC-MS. Todas las corridas fueron conducidas a través de columnas capilares $(60 \mathrm{~m}, 0.25 \mathrm{~mm}$ ID, $0.25 \mu \mathrm{m}$ grosor del film) Elite WAX de sílica fundida Perkin Elmer, utilizando Helio como gas conductor (49.6 psi). Se seleccionó el modo de inyección "splitless". La ionización se llevó a cabo en un espectrómetro de masas al vacío por impacto de electrones con una energía de ionización 70 eV-.

Las muestras se analizaron utilizando el siguiente protocolo: Temperatura inicial de $40^{\circ} \mathrm{C}$ (durante 3 min), $10^{\circ} \mathrm{C} / \mathrm{min}$ a $240^{\circ} \mathrm{C}$ (durante 10 minutos). Se utilizó una presión en cabeza de columna de 15 psi y una temperatura del inyector de $250{ }^{\circ} \mathrm{C}$. La línea de transferencia de GC se mantuvo a $200^{\circ}$ C. Los cromatogramas se adquirieron en modo de exploración escaneando el cuadrupolo de $\mathrm{m}=\mathrm{z} 50$ y $\mathrm{m}=\mathrm{z} 300$ (tiempo de exploración: 0,2 s, tiempo de inter-scan: $0,1 \mathrm{~s})$.

Para verificar la inocuidad del aceite mineral sobre el patrón visual de la corona se procedió a cortar los filamentos de los radii y agruparlos según su color (azulado, blanco y morado) para aumentar la superficie de incidencia del haz de luz del espectrofómetro de reflexión (Ocean optics USB4000). Los espectros fueron analizados con el programa Ocean Spectra Suite.

\section{Estudios de preferencia: Función visual (Tratamiento T1)}

Para comprobar la función visual de la corona, fue necesario anular la función odorífera. Una vez comprobada la efectividad del aceite mineral para esta tarea, se pintó con dicha sustancia los radii de 15 flores de al menos 3 individuos, para impedir de esta manera la emisión de los compuestos volátiles. El control de este tratamiento $(\mathrm{C} 1)$ se realizó con 


\section{G. Varela et al. - Función de la corona en Passiflora caerulea}

otras 15 flores intactas y sin aplicación de aceite mineral.

\section{Estudios de preferencia: Función odorifera (Tratamiento T2)}

Para comprobar la función odorífera de la corona se anuló la función visual, para ello se cubrió la corona de 15 flores de al menos 3 individuos con piezas periánticas de otras flores de la misma especie (Fig. 2, A y B), permitiendo la emisión de los aromas de la corona. Como control (C2), para otras 15 flores se colocaron las piezas periánticas extras por debajo del propio perianto de la flor control (Fig. 2, C y D), esto es para controlar el efecto de olor de corte si lo hubiera, reproducir el mismo efecto de contorno que presentan las flores tratadas al agregarle las piezas periánticas y producir la misma intensidad de aroma del perianto.

\section{Estudios de preferencia: Función visual-olfativa (Tratamiento T3)}

Para verificar la función visual-odorífera de la corona se removieron los radii en 15 flores de al menos 3 individuos, de manera de suprimir ambos tipos de atracción (Fig. 2, E). Como control de este tratamiento $(\mathrm{C} 3)$ se ofrecieron a los polinizadores a la par de cada flor tratada otra con la corona intacta (Fig. 2, F).

Para todos los tratamientos (T1, T2, y T3) se registraron tanto la frecuencia de visitas, así como las aproximaciones a las flores tratadas, ya que éstas ofrecían características atípicas que no les permitían a los polinizadores posarse normalmente (radii cubiertos con aceite o ausencia de radii), completando un total de 100 visitas entre cada par de flores. Las flores de cada par (tratada-control) se encontraban a una distancia no menor a un metro.

Por tratarse de datos de frecuencia se analizaron todos los tratamientos (T1, T2, T3) mediante Modelo Lineal Generalizado (GLM) con enlace de tipo Poisson, para determinar si existían diferencias significativas dentro de cada tratamiento respecto de su control y entre los tratamientos.

\section{Resultados}

Experiencias sobre la emisión de aromas florales y el efecto supresor del aceite en su volatilización

Se obtuvieron cinco cromatogramas (Fig. 3 A:
Perianto, B: Radii, C: Radii con aceite mineral, D: Control ambiental, E: Control aceite mineral). Las cromatografías para los radii tratados con aceite y los controles ambiental y del aceite (Fig 3 D y E) no evidenciaron la presencia de ningún compuesto volátil. Por otra parte, se observó la presencia de picos en los detalles de los cromatogramas para el perianto (Fig.4 A) y de los radii sin tratamiento (Fig.4 B), siendo los compuestos hallados: (a) $\alpha$-Copaeno, (b) 2,6-Farnesol y (c) D-Germacreno para el perianto y (d) (-)- $\alpha$-Cubebeno y (e) MetilLinolenato para los radii. También se observaron picos pronunciados (como los del tiempo 23,9 o 27,1) que; por hallarse presentes en todas las cromatografías, incluyendo los controles; no se tuvieron en cuenta para el análisis y se interpretaron como errores de lectura.

Por otra parte, los espectros obtenidos para cada color de los radii (morado, blanco y azulado) y para los mismos colores tratados con aceite mineral (Fig.5) revelan que en general las formas de las curvas en cada par se mantienen sin grandes modificaciones, es decir que la calidad del color en cuanto al rango del espectro reflejado no se ve afectada por el uso del aceite mineral, sólo se detectó una leve reducción de la intensidad de reflexión en las flores tratadas con aceites.

\section{Estudios de preferencia:}

Según las especificaciones mencionadas anteriormente se obtuvo un registro de las visitas para los distintos tratamientos y sus respectivos controles para cada tratamiento (Tabla 1).

En todos los tratamientos hubo una reducción significativa en la frecuencia de visitas con respecto a los respectivos controles (Tabla 2, Fig. 6). Los modelos lineales generalizados usados para la comparación entre tratamientos mostraron diferencias significativas entre los tratamientos $\mathrm{T} 1 \mathrm{y}$ $\mathrm{T} 2$ con el T3, mientras que no fueron significativas entre los tratamientos T1 y T2 (Tabla 3, Fig. 6).

\section{Función visual (Tratamiento T1)}

En muchos casos las abejas y abejorros revoloteaban cerca de las flores pero su aterrizaje sobre ellas se veía dificultado debido a la presencia del aceite sobre los radii. Se observó que en los casos en que lograban posarse, el tiempo de permanencia en las flores tratadas fue mucho menor que en las flores control. 
Bol. Soc. Argent. Bot. 51 (1) 2016
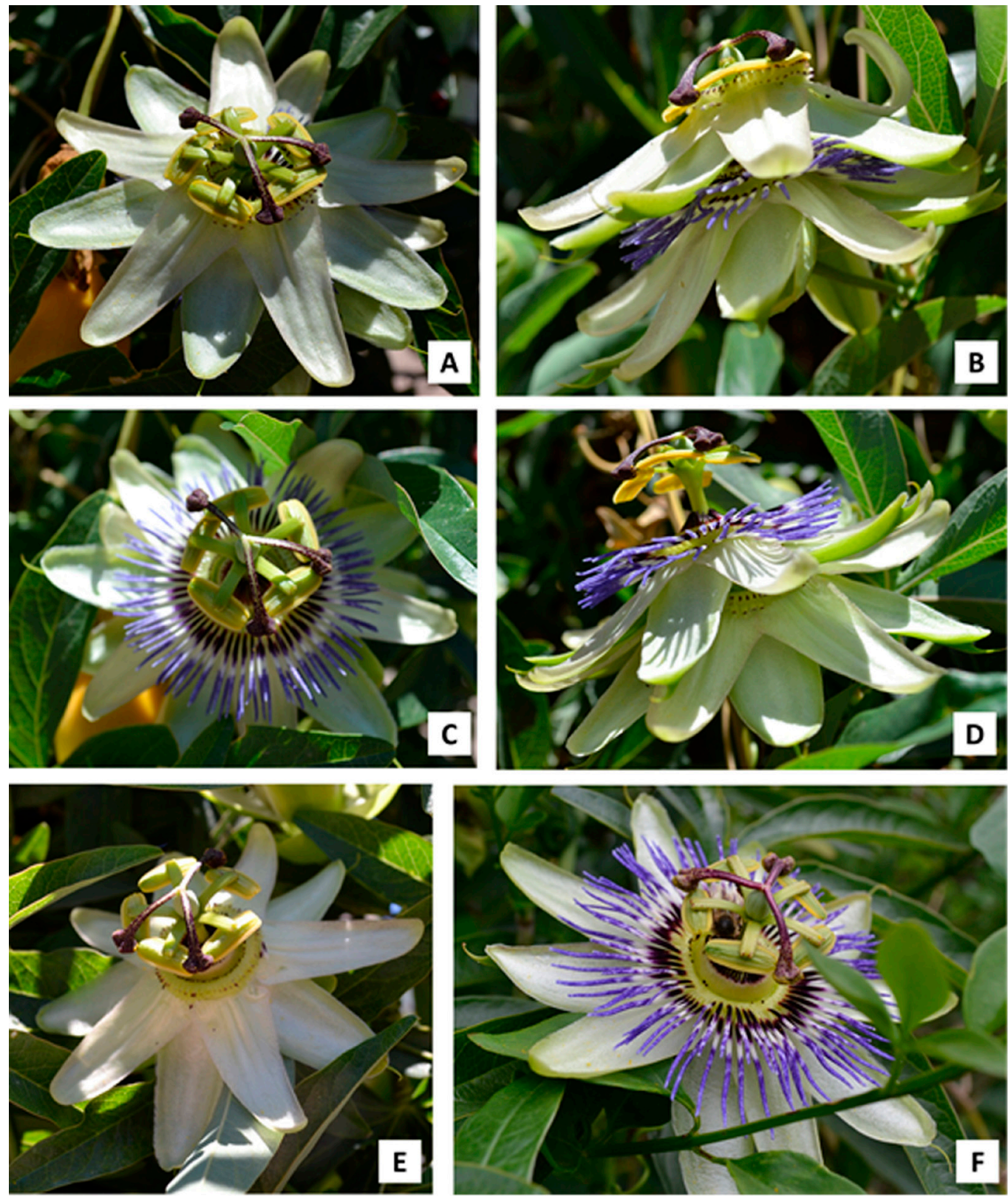

Fig. 2. A: Vista superior. B: Lateral de una flor correspondiente al tratamiento T2. C: Vista superior. D: Lateral de una flor correspondiente al control C2. E: Vista superior de una flor correspondiente al tratamiento T3. F: Vista superior de una flor correspondiente al control C3.

Función odorífera de la corona (Tratamiento T2)

La mayoría de los agentes que se posaban en las flores no alcanzaron la cámara nectarífera con sus piezas bucales, exceptuando algunos individuos de Apis mellifera que, por su pequeño tamaño, se introducían por los espacios libres entre las piezas extra y las propias de las flores.

Función visual-olfativa de la corona (Tratamiento T3)

Si bien las flores estaban desprovistas de los radii, las aproximaciones en general terminaron en visitas, ya que los polinizadores se posaban sobre el perianto. 

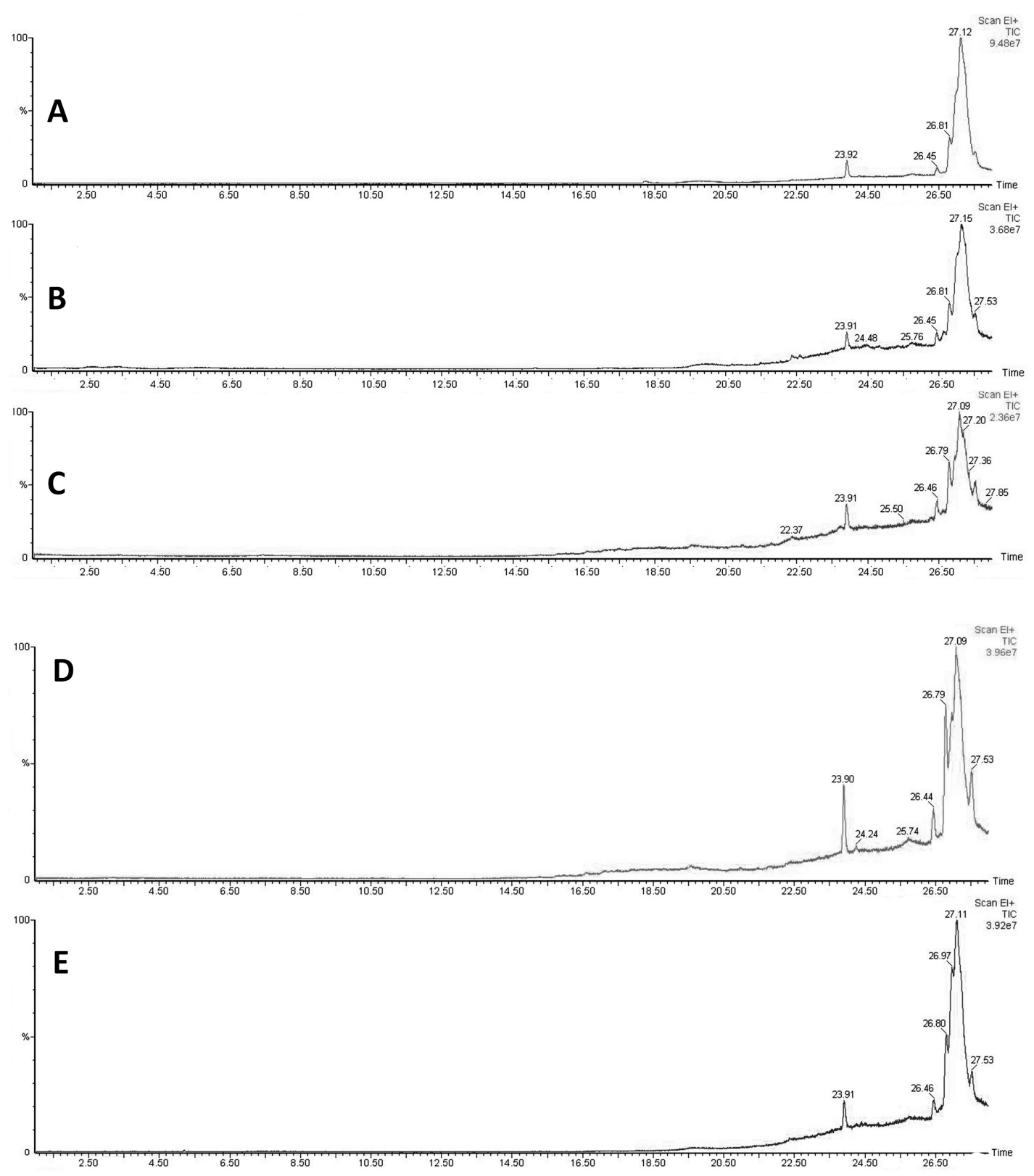

Fig. 3. Cromatografías para (A) el perianto, (B) los radii de la corona, (C) los radii tratados con aceite mineral, y para los controles (D) ambiental y (E) del aceite.

\section{Discusión}

El uso de aceite mineral resultó apropiado para realizar los tratamientos en los ensayos de frecuencias de visitas para probar la función visual (T1), ya que no produjo ningún aroma adicional y las modificaciones visuales no resultaron relevantes para los insectos, que no utilizan el brillo o reflectancia para distinguir los colores (Newman et al., 2012).

Mediante ensayos experimentales se ha podido evaluar la función de los ciclos más externos de la corona de $P$. caerulea como atrayente, tanto visual como odorífera. 


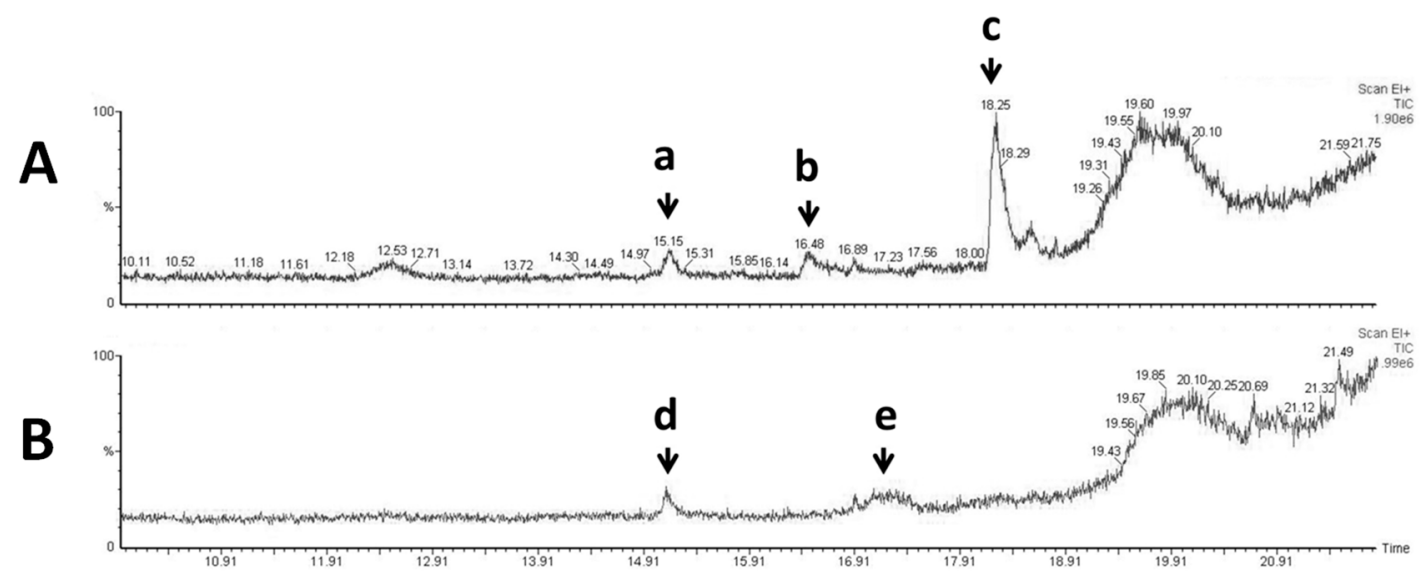

Fig. 4. Detalles de las cromatografías $A$ y $B$ de la Figura 3, mostrando los picos correspondientes a los compuestos volátiles encontrados: (a) a-Copaeno, (b) 2,6-Farnesol (c) D-Germacreno, (d) (-)-a-Cubebeno y (e) Metil-Linolenato.

Ambas funciones postuladas se comprobaron, y de ellas la emisión de aromas resultó ser la más relevante, ya que la disminución en la frecuencia de visitas resultó ser similar tanto cuando se eliminaba
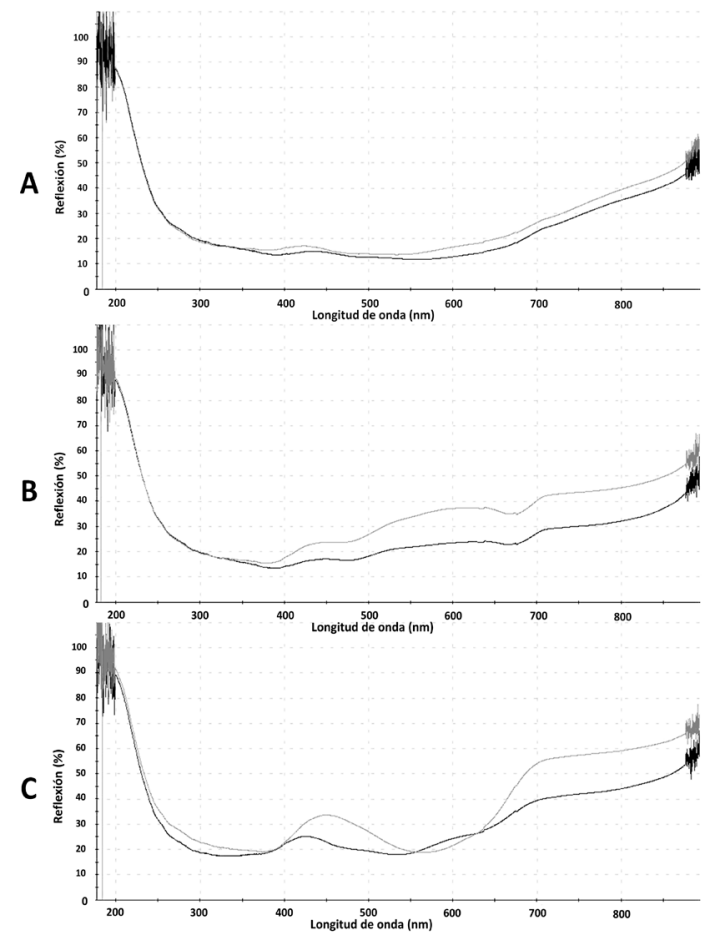

Fig. 5. Espectrometrías para los distintos colores de los filamentos de la corona (A) Morado, (B) Blanco y (C) Azulado; tratados con aceite mineral (línea en negro) y sin tratar (línea en gris). esta función (T1), como cuando se eliminaban de manera completa los radii (T3). En cambio, cuando se suprimía el efecto visual (T2), hubo una disminución en la frecuencia de visitas respecto de su control, pero significativamente menor que en los tratamientos anteriores. Esto podría deberse a que las abejas y abejorros, así como muchos otros polinizadores utilizan los aromas como señales clave para reconocer sus recursos alimenticios (Harborne, 1988; Rieger \& Jacob, 1988) permitiendo su localización a larga distancia (Dudareva \& Pichersky, 2000), mientras que la visión sería utilizada para reconocer las flores entre el follaje, a distancias más cortas (Galarda Varassin et al., 2001).

Antecedentes previos reportaron que la producción de aromas se producía en diferentes partes de la corona, y que era más intensa en los radii (MacDougal, 1994; Amela García, 1999); por otro lado se localizaron los tejidos productores de las fragancias por medio de estudios anatómicos (Amela García et al., 2007), pero no se había reportado aún la producción de aromas por parte de las piezas periánticas. En este trabajo se demuestra que tanto los radii como las piezas periánticas producen fragancias y que los aromas producidos por ambas estructuras difieren en su composición química, perteneciendo a dos grandes familias según su naturaleza química. Sesquiterpenos: $\alpha$-Copaeno; 2,6-Farnesol; D-Germacreno y (-)- $\alpha$-Cubebeno y Ésteres: Metil-linolenato. Estos compuestos 
Tabla 1. Cantidad de visitas de abejas y abejorros sobre los quince pares de flores tratadas-control de $P$. caerulea analizadas para comprobar las funciones visual y odorífera de la corona. Se contabilizaron las visitas siempre hasta llegar a un total de 100 entre el par de flores tratadas-control.

\begin{tabular}{|cccccc|}
\hline $\begin{array}{c}\text { TRATAMIENTO } 1 \\
\text { (Sin función odorifera) }\end{array}$ & \multicolumn{2}{c}{$\begin{array}{c}\text { TRATAMIENTO } 2 \\
\text { (Sin función visual) }\end{array}$} & \multicolumn{2}{c|}{$\begin{array}{c}\text { TRATAMIENTO 3 } \\
\text { (Sin función visual-odorífera) }\end{array}$} \\
\hline T1 & Control (C1) & T2 & Control (C2) & T3 & Control (C3) \\
\hline 9 & 91 & 28 & 72 & 12 & 88 \\
12 & 88 & 29 & 71 & 17 & 83 \\
15 & 85 & 31 & 69 & 17 & 83 \\
17 & 83 & 31 & 69 & 18 & 82 \\
18 & 82 & 32 & 68 & 21 & 79 \\
18 & 82 & 32 & 68 & 22 & 78 \\
18 & 82 & 33 & 67 & 26 & 74 \\
\hline 19 & 81 & 34 & 66 & 26 & 74 \\
\hline 19 & 81 & 35 & 65 & 27 & 73 \\
\hline 20 & 80 & 37 & 63 & 28 & 72 \\
\hline 22 & 78 & 37 & 63 & 29 & 71 \\
\hline 23 & 77 & 39 & 61 & 30 & 70 \\
\hline 28 & 72 & 42 & 58 & 30 & 70 \\
\hline 33 & 67 & 43 & 57 & 32 & 68 \\
\hline 33 & 67 & 44 & 56 & 47 & 53 \\
\hline
\end{tabular}

Tabla 2. Valores $Z$ y p para cada Tratamiento $(T)$ y su respectivo Control $(\mathrm{C})$ correspondientes a cada prueba para las funciones de la corona (T1, T2 y T3).

\begin{tabular}{|ccccc|}
\hline & & & $\mathbf{Z}$ & $\boldsymbol{P}$ \\
T1 & vs. & C1 & 21,32 & $<2 \mathrm{e}-16$ \\
T2 & vs. C2 & 11,34 & $<2 \mathrm{e}-16$ \\
T3 & vs. & C3 & 63,27 & $<2 \mathrm{e}-16$ \\
\hline
\end{tabular}

difieren de los encontrados por Galarda Varassin et al. (2001) para Passiflora alata. Esta especie, también melitófila, presentó otros ésteres entre los compuestos responsables del aroma floral (Benzoato de bencilo y Methyl 9-hexandecenoato) y ningún sesquiterpeno, aunque sí otros terpenoides (Linalol, Nerol, Geraniol y Citral). Esto sugiere la singularidad y complejidad en la composición de los aromas florales para cada especie, aunque estén representados los mismos grupos o familias de compuestos químicos. De cualquier manera, aunque no se conoce mucho sobre cómo los insectos responden a los componentes individuales, es claro que estos animales son capaces de distinguir entre mezclas complejas de aromas florales (Dudareva \& Pichersky, 2000).

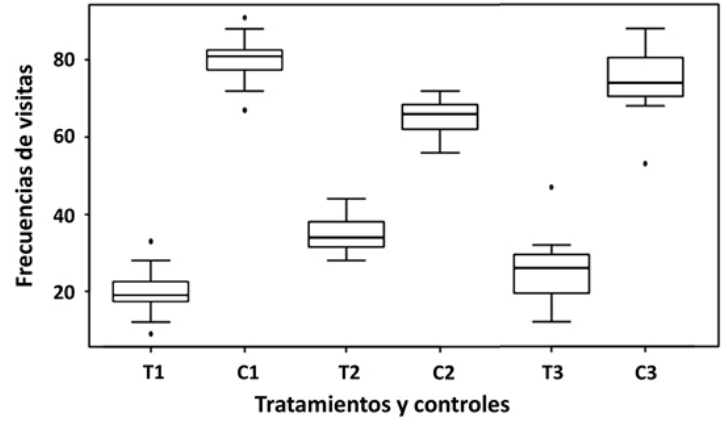

Fig. 6. Boxplot para las frecuencias de visitas entre todos los tratamiento (T1- T2 -T3) y dentro de cada tratamiento (T1-C1, T2-C2, T3-C3) para un total de 100 visitas en cada muestra.

Tabla 3. Valores $F$ y $P$ entre todos los posibles pares de tratamientos (T1-T3, T1-T2 y T2- T3).

\begin{tabular}{|ccccc|}
\hline & & & $\boldsymbol{F}$ & $\boldsymbol{P}$ \\
T1 & vs. & T3 & 18,05 & 0,108 \\
T1 & vs. & T2 & 18,05 & $1,5 \mathrm{e}-6$ \\
T2 & vs. & T3 & 18,05 & 0,001 \\
\hline
\end{tabular}


Por otra parte, los compuestos encontrados en $P$. caerulea en este trabajo, excepto el Metil linolenato, están citados por Knudsen et al. (2006) como responsables del aroma floral en Passifloraceae.

Investigaciones recientes indican que la emisión de compuestos volátiles por parte de las plantas depende de la edad de la flor, hora del día, temperatura y humedad relativa del ambiente, temperatura de la flor, su exposición al sol y si ha sido polinizada o no (Azuma et al., 1999; Terry et al., 2004; Niemeyer \& Teiller, 2007). Un posible estudio posterior podría abordar estas cuestiones sobre $P$. caerulea.

Si bien los resultados de este trabajo indicaron que la eliminación de la función visual no fue tan importante en cuanto a la reducción de la frecuencia de visitas como cuando se eliminó la producción de fragancias, el atractivo visual puede contribuir al atractivo odorífero. Con respecto a esta función de la corona, Amela García \& Hoc (1997) describieron un patrón de reflexión y absorción UV de anillos concéntricos en $P$. caerulea que funcionaría como guías de néctar que, según Vogel (1990), coinciden con guías de aroma y en conjunto indicarían a los insectos dónde se encuentra la recompensa (Free, 1970; Kevan, 1983). Además de estos patrones de coloración de las propias flores, es importante el contraste con el fondo de modo que los agentes de polinización, como los abejorros, encuentren sus fuentes de recompensa (Spaethe et al., 2001), incrementando el número de visitas exitosas que lleven a un aumento del éxito reproductivo de la planta (Chittka, 1996). Amela García (1999) interpretó que el escaso contraste con el fondo de las flores de Passiflora estaría compensado con el patrón de círculos concéntricos, ya que constituye una figura de gran intensidad según Barth (1991), citado en Amela García (1999).

\section{Conclusiones}

Se verificó la importancia de la corona como atrayente de los vectores de polinización en $P$. caerulea mediante la comparación de los resultados de cada tratamiento con su control. En todos los casos la aplicación del tratamiento disminuyó la cantidad de visitas con respecto a su control en cantidades significativas, esta disminución fue mayor cuando se anuló la función odorífera (T1) y la visual-odorífera (T3), que cuando se anuló solo la función visual (T2), denotando la menor importancia de esta última. Adicionalmente, se comprobó que suprimir la emisión de aromas de los radii produce un efecto similar en la cantidad de visitas al de removerlos por completo $(\mathrm{T} 1=\mathrm{T} 3)$, lo que en conjunto indica que en $P$. caerulea la emisión de aromas es la función más efectiva de los radii, y por extensión de la corona, para la atracción de los polinizadores.

Por otra parte, se identificaron los compuestos responsables del aroma floral, cuatro sesquiterpenos: $\alpha$-Copaeno, 2,6-Farnesol, D-Germacreno y (-)- $\alpha$-Cubebeno, y un éster: Metil-Linolenato, los tres primeros secretados por el perianto y los dos últimos por los radii, demostrando que los aromas florales no solo se secretan en los radii, como se ha indicado anteriormente, sino también en los ciclos del perianto. Queda como futura posibilidad de estudio discernir si son solamente los radii, en conjunto con otro ciclo, o todos los ciclos de la corona los responsables de atraer a los vectores de polinización.

\section{Agradecimientos}

A la Dra. Marcela Palacio, del INFICQ-IMBIV (CONICET-UNC), por su ayuda en la asistencia en el análisis de GCMS, a los Dres. Santiago BenítezVieyra y Marcela Moré, ambos pertenecientes al laboratorio de Ecología Evolutiva y Biología Floral (FCEFyN-UNC, IMBIV-CONICET) por sus aportes sobre la metodología y el análisis de resultados.

\section{Bibliografía}

AMELA GARCÍA, M. T. 2013. Intra-specific floral variability among Passiflora species in Argentina. Acta Hort. 1000: 157-164.

AMELA GARCÍA, M. T. 1999. Biología floral y sistema reproductivo de especies nativas de Passiflora (Passifloraceae) de la Argentina. Tesis de doctorado, Universidad de Buenos Aires, Argentina.

AMELA GARCÍA, M. T., \& P. S HOC. 1997. Floral biology and reproductive system of Passiflora caerulea (Passifloraceae). Beitr. Biol. Pflanz. 70: 1-20.

AMELA GARCÍA, M. T. \& P. S. HOC. 2012. Pollination mechanisms in Passiflora species: the common 


\section{G. Varela et al. - Función de la corona en Passiflora caerulea}

and the rare flowers. Ecological aspects and implications for horticulture. En: RASKIN, N. D. \& P. T. VUTURRO (eds.), Pollination: Mechanisms, Ecology and Agricultural Advances, pp 33-55. Nova Science Publishers, New York.

AMELA GARCÍA, M. T., B. G GALATI \& P. S. HOC. 2007. Ultrastructure of the corona of scented and scentless flowers of Passiflora spp (Passifloraceae). Flora 202: 302-315.

AZUMA, H., L. B. THIEN, \& S. KAWANO. 1999. Floral scents, leaf volatiles and thermogenic flowers in Magnoliaceae. Plant. Spec. Biol. 14: 121-127.

CHITTKA, L. 1996. Optimal sets of color receptors and color opponent systems for coding of natural objects in insect vision. J. Theor. Biol. 181: 179-196.

CUSSET, G. 1967. Les passifloracees asiatiques. Adansonia 7: 371-385.

DAFNI, A. 1992. Pollination ecology: a practical approach. Oxford University Press, Oxford.

DEGINANI, N. B. 2001. Las especies argentinas del género Passiflora (Passifloraceae). Darwiniana 39: 43-129.

DHAWAN, K., S. DHAWAN \& A. SHARMA. 2004. Passiflora: a review update. J. Ethnopharmacol. 94: $1-23$.

DOBSON, H. E., J. ARROYO, G. BERGSTRÖM \& I. GROTH. 1997. Interspecific variation in floral fragrances within the genus Narcissus (Amaryllidaceae). Biochem. Syst. Ecol. 25: 685-706.

DUDAREVA, N. \& E. PICHERSKY. 2000. Biochemical and molecular genetic aspects of floral scents. Plant. Physiol. 122: 627-634.

ENDRESS, P. K. \& M. L. MATTHEWS. 2006. Elaborate petals and staminodes in eudicots: diversity, function, and evolution. Org. Divers. Evol. 6: 257-293.

FAEGRI, K. \& L. VAN DER PIJL. 1979. The principles of pollination ecology. 3ra ed. Pergamor press, Oxford.

FREE, J. B. 1970. Insect pollination of crops. Academy Press, London.

GALETTO, L. 2006. Morfología y anatomía floral en especies de Apocynaceae-Asclepiadoidea. Kurtziana 32: 5-11.

GALARDA VARASSIN, I., J. R., TRIGO, \& M., SAZIMA. 2001. The role of nectar production, flower pigments and odour in the pollination of four species of Passiflora (Passifloraceae) in southeasthern Brazil. Bot. J. Linn. Soc. 136, 139-152.

GÓMEZ, J. M. 2002. Generalización en las interacciones entre plantas y polinizadores. Rev. Chil. Hist. Nat. 75: 105-115.

HARBORNE, J. B. 1988. Biochemistry of plant pollination. En: HARBORNE, J. B. (eds.), Introduction to ecological biochemistry, pp. 42-81. Academic Press, London.
HEMINGWAY, C. A., A. R. CHRISTENSEN \& S. T. MALCOMBER. 2011. B-and C-class gene expression during corona development of the blue passionflower (Passiflora caerulea, Passifloraceae). Am. J. Bot. 98: 923-934.

HICKEY, M. \& C. KING. 1988. 100 Families of Flowering Plants. 2da ed. Cambridge University Press, Cambridge.

HODGINS, K. A. \& S. C. BARRETT. 2008. Natural selection on floral traits through male and female function in wild populations of the heterostylous daffodil Narcissus triandrus. Evolution 62: 17511763.

JANZEN, D. H. 1968. Reproductive behavior in the Passifloraceae and some of its pollinators in Central America. Behaviour 32: 33-48.

KEVAN, P.G. 1983. Floral colors through the insect eye: what they are and what they mean. En: JONES, C.E. \& R.J. LITTLE (eds.), Handbook of experimental pollination biology, pp 3-30. Van Nostrand Reinold, New York.

KILLIP, E. P. 1938. The American species of Passifloraceae. Field Mus. Nat. Hist., Bot. Ser. 19: 1-613.

KNIGHT Jr, R. J. \& H. F. WINTERS. 1962. Pollination and fruit set of yellow passion fruit in southern Florida. P. Fl. St. Hortic. Soc. 75: 412-418.

KNUDSEN, J. T., R. ERIKSSON, J. GERSHENZON \& B. STÅHL. 2006. Diversity and distribution of floral scent. Bot. Rev. 72: 1-120.

LINDBERG, A. B., J. T. KNUDSEN \& J. M. OLESEN. 2000. Independence of floral morphology and scent chemistry as trait groups in a set of Passiflora species. En: TOTLAND, O. (eds.), The Scandinavian Association for Pollination Ecology honours Knut Fægri. Det Norske Videnskaps-Akademi. I. Mat.Naturv. Klasse, Ny Ser. 39: 91-111.

LINDMAN, C. A. M. 1906. A vegetação no Rio Grande do Sul (Brasil austral). Livraria Universal, São Paulo, Belo Horizonte.

MACDOUGAL, J. M. 1994. Revision of Passiflora subgenus Decaloba section Pseudodysosmia (Passifloraceae). Sys. Bot. Monogr. 41: 1-146.

MAOUT, L.E. \& J. DECAISNE. 1876. A General System of Botany. Longmans, Green and Company, London.

MENZEL, R., U. GREGGERS \& M. HAMMER. 1993. Functional organization of appetitive learning and memory in a generalist pollinator, the honey bee. En: PAPAJ, D., LEWIS, A. C. (eds.), Insect learning: ecological and evolutionary perspectives. pp 79-125. Chapman \& Hall, New York.

NEWMAN, E., B. ANDERSON, \& S. D. JOHNSON .2012. Flower color adaptation in a mimetic orchid. $P$. Roy. Soc. B-Biol. Sci. 279: 2309-2313.

NIEMEYER, H. M. \& S. TEILLIER. 2007. Aromas de la flora nativa de Chile. Universidad de Chile, Chile. 
OLSEN, K. M. 1997. Pollination effectiveness and pollinator importance in a population of Heterotheca subaxillaris (Asteraceae). Oecologia 109: 114-121.

PURI, V. 1948. Studies in floral anatomy, V. On the structure and nature of the corona in certain species of the Passifloraceae. J. Ind. Bot. Soc. 27: 130-149.

RENDLE, A. B. 1959. Classification of Flowering Plants. Cambridge University Press, Cambridge.

RIEGER, J. F. \& E. M. JAKOB. 1988. The use of olfaction in food location by frugivorous bats. Biotropica 20: 161-164.

SMITH, B. H. \& W. M. GETZ .1994. Nonpheromonal olfactory processing in insects. Annu. Rev. Entomol. 39: 351-375.

SPAETHE, J., J. TAUTZ \& L. CHITTKA. 2001. Visual constraints in foraging bumblebees: flower size and color affect search time and flight behavior. P. Natl. Acad. Sci. USA. 98: 3898-3903.

STEBBINS, G. L. 1970. Adaptive radiation of reproductive characteristics in angiosperms, I: Pollination mechanisms. Annu. Rev. Ecol. Syst. 1: 307-326.

STEVENS, P. F. 2001. onward. Angiosperm Phylogeny Website. Version 9, June 2008 (and more or less continuously updated). Disponible en: http: /www. mobot.org /MOBOT /research/APweb/ [Acceso: Diciembre 2015].

TERRY, I., C. J. MOORE, G. H. WALTER, P. I.
FORSTER, R. B. ROEMER, J. D. DONALDSON \& P. J. MACHIN. 2004. Association of cone thermogenesis and volatiles with pollinator specificity in Macrozamia cycads. Plant Syst. Evol. 243: 233-247.

TORRES, C., J.S. DAMBOLENA, M.P. ZUNINO \& L. GALETTO. 2012. Nectar characteristics and pollinators for three native co-occurring insect pollinated Passiflora (Passifloraceae) from central Argentina. Int. J. Plant. Reprod. Biol. 4: 121-126.

ULMER, T. \& J. M. MACDOUGAL. 2004. Passiflora: passionflowers of the world. Timber Press, Portland, Oregon.

VOGEL, S. 1990. The role of scent glands in pollination: on the structure and function of osmophores. Amerind Publishing Company, New Delhi, India.

YOCKTENG, R. \& S. NADOT. 2004. Phylogenetic relationships among Passiflora species based on the glutamine synthetase nuclear gene expressed in chloroplast (ncpGS). Mol. Phylogenet. Evol. 31: 379-396.

ZHANG, Z. \& J. PAWLISZYN. 1993. Headspace solidphase microextraction. Anal. Chem. 65: 1843-1852.

Recibido el 2 de febrero de 2015, aceptado el 25 de noviembre de 2015 . 